ACTA UNIVERSITATIS LODZIENSIS

Folia Litteraria Romanica 16, 2021

https://doi.org/10.18778/1505-9065.16.15

Agnieszka Konowska

Université de Łódź

(iD) https://orcid.org/0000-0002-3041-869X

agnieszka.konowska@uni.lodz.pl

\title{
La fonction argumentative de la doxa dans le discours sur le couple Macron
}

\begin{abstract}
RÉSUMÉ
En analysant quelques échantillons du discours numérique sur le couple présidentiel français, cet article a pour objectif de déterminer comment certains stéréotypes concernant l'amour intergénérationnel sont convoqués de manière à argumenter contre la politique d'Emmanuel Macron. Les couples où la femme est bien plus âgée que son partenaire s'opposent à la doxa ambiante qui tient pour naturelle une relation où c'est le contraire. Les époux Macron sont ainsi stigmatisés et deviennent la cible de discours discriminants visant principalement Brigitte Macron, attaquée pour son âge et son physique. La thèse est que le recours aux stéréotypes relève d'une argumentation $a d$ personam qui a pour but de discréditer le président de la République. Le caractère « anti-doxique» de sa relation avec Brigitte, mis au premier plan par ses antagonistes, agit comme un instrument de délégitimation de sa personne et, chemin faisant, de sa politique. Dans le cas analysé, l'argumentation ad personam s'effectue à travers une démarche toute singulière que l'on pourrait appeler ad hoc une argumentation « ad coniugem ».
\end{abstract}

MOTS-CLÉS - stéréotype, doxa, argumentation dans le discours, stigmatisation, relation

\section{The Argumentative Function of the doxa in the Discourse on the Macron Couple}

\section{SUMMARY}

By analyzing a variety of samples of the digital discourse on the French presidential couple, the aim of this article is to determine how certain stereotypes concerning intergenerational love are invoked in order to argue against the policy of Emmanuel Macron. Couples with a significant age difference in favor of women violate current doxa, because the relationship where the man is older is "normal" or "imaginable". The Macrons are thus stigmatized and become the target of discriminatory discourse which mainly attacks the president's wife because of her age and physical appearance. The thesis is that the mentioned stereotypes are mobilized for ad personam argumentation which aims to discredit the President of the Republic. The "anti-doxic" character of his relationship with Brigitte, put in the foreground by his antagonists, acts as an instrument of delegitimization of his person and, in the process, of his policy. In the analyzed case, the ad personam argumentation is carried out in a very peculiar way that one could call ad hoc an "ad coniugem" argumentation.

KEYWORDS - stereotype, doxa, argumentation in discourse, stigmatization, relationship 


\section{Introduction}

Parmi les multiples facteurs à prendre en compte dans l'analyse argumentative du discours (Amossy, 2006), s'il en est un incontournable, c'est bien la doxa ${ }^{1}$ et ses manifestations, les stéréotypes ${ }^{2}$. Le présent article se propose d'examiner le rôle des préjugés concernant l'amour intergénérationnel (vécu par les couples à grande différence d'âge) dans les stratégies argumentatives mises en place par les opposants politiques d'Emmanuel Macron, de 24 ans le cadet de son épouse. Les couples à l'écart d'âge important en faveur de la femme s'opposent à la doxa qui tient pour naturelle une relation où l'homme est plus âgé que sa partenaire (les deux devant être de préférence issus d'une même génération), ainsi le couple présidentiel est-il vu comme atypique, contre nature, « anti-doxique».

Le recours à cette doxa stigmatisante pour discréditer E. Macron relève d'une argumentation ad personam, qui consiste « en une mise en cause frontale de sa personne plutôt qu'en une opposition à son point de vue » (Gauthier, 2011 : 87), à ses idées ou à sa politique. Il va sans dire qu'il s'agit alors d'une argumentation fallacieuse visant à décrédibiliser l'adversaire, se résumant très souvent à des coups bas, des généralisations abusives et des insultes. Dans le cas analysé, c'est l'épouse du président qui en devient la cible, on est donc en présence d'une stratégie que l'on pourrait nommer « ad coniugem ».

Dans un premier temps, nous nous pencherons sur les principales idées reçues concernant le couple pour montrer comment les Macron vont à l'encontre de certaines normes sociales tacites, mais bien connues et inconsciemment partagées par une grande partie de la société française. Comme on s'en prend surtout

1 Entendue comme «l'opinion commune dans une société donnée à une époque donnée » (Angenot, 2013 : 44), « un fonds d'idées, voire de préjugés qui, consciemment ou inconsciemment, représentent la mentalité d'une communauté » (Schapira, 1999 : 32), « le savoir partagé d'une communauté à une époque donnée » (Amossy, 2006 : 104).

2 Tout au long de cet article, nous allons utiliser les termes stéréotype, idée reçue et préjugé, lesquels, pour proches qu'ils soient, ne doivent pas être amalgamés. Suivant Amossy (2006 : 121), nous entendons par stéréotype, " une représentation ou une image collective simplifiée et figée des êtres et des choses que nous héritons de notre culture, et qui détermine nos attitudes et nos comportements ». Il est « un élément doxique obligé sans lequel [...] aucune opération de catégorisation ou de généralisation ne serait possible » (p. 122). La notion d'idée reçue met l'accent sur le caractère prescriptif des opinions partagées : comme le dit Slakta (1994: 43), « une idée reçue, c'est toujours une idée à recevoir ; à mettre en pratique ». En rapport étroit avec l'autorité et l'injonction sociale, les idées reçues sont « le prêt-à-dire, le prêt-à-penser, le prêt-à-faire, prescrit par le discours social » (Amossy et Herschberg Pierrot, 1997 : 24). Pour ce qui est de la différence entre préjugé et stéréotype, nous retiendrons la vision des linguistes qui viennent d'être évoquées : «[...] le stéréotype apparaît comme une croyance, une opinion, une représentation concernant un groupe et ses membres, alors que le préjugé désigne l'attitude adoptée envers les membres du groupe en question. Harding voit dans le préjugé "une attitude envers les membres d'un groupe extérieur où les tendances à l'évaluation négative prédominent" (Harding, 1968) [...] » (p. 34-35, le gras est des auteures). 
à l'épouse du président, il sera nécessaire d'évoquer, par la suite, les images de la femme ancrées dans la doxa française et ce qui en émerge de façon concrète, à savoir les préjugés sexistes qui inspirent les insultes envers Brigitte Macron. Finalement, nous verrons comment, d'une manière consciente ou non, les antagonistes du président, journalistes et citoyens ordinaires, mobilisent certaines opinions répandues et évidences culturelles pour délégitimer sa personne.

\section{Les idées reçues sur le couple « modèle »}

\subsection{Le couple « modèle " : la même génération}

Le couple présidentiel déroge clairement à l'idée reçue qui impose l'appartenance des partenaires à la même génération. Ceci se reflète dans les médias qui parlent des Macron en termes de « couple atypique » (Le Point, Maris Match), « inhabituel » (Courrier international), « original » (Terra femina), « peu ordinaire » (TV5 Monde), « pas comme les autres » (Marie Claire), « singulier » (Gala), « disruptif » (L'Express), « hors du commun »(Le Nouvel Observateur) ou « hors-norme » (Sud-Ouest).

\subsection{Dans le couple, l'homme devrait être plus âgé que la femme}

La société voit d'un meilleur œil que l'homme soit plus âgé que sa partenaire : l'idée généralement partagée est que la femme jouit de la protection masculine et non l'inverse ${ }^{3}$. B. Macron l'emportant sensiblement par l'âge, la critique du caractère anti-doxique de cette relation s'étend dans deux directions : soit l'on a affaire au mépris et aux insultes concernant l'âge de la femme, soit l'authenticité du couple est contestée.

Dans le premier cas, le discours sur l'âge de B. Macron foisonne d'insultes contextuelles et directes. Elle est réduite à une seule de ses dimensions, devenant « la première vieille dame de France », « un monument historique », « Momie Bribri » ou « Notre Dame de l'Élysée ». Les insultes sont souvent implicites et nécessitent une interprétation plus subtile, comme p. ex. la réaction caustique d'un(e) internaute aux paroles émues de B. Macron après l'incendie de NotreDame : "C'est un message qu'elle [la cathédrale] nous envoie. Je le sais ». L'internaute lance : " Entre ruines elles communiquent et se comprennent » (Huguette Lagarde sur sputniknews.com). Un(e) autre demande malicieusement si la femme du président sera « installée au Louvre » (Sylvain Treffé sur madame. lefigaro.fr).

${ }^{3}$ Cf. p. ex. M. Bozon (1990, 1991), J. Coenen-Huther (2001), J.-F. Mignot (2010). 
Certaines insultes contextuelles consistent à nier son rôle d'épouse en l'identifiant à la mère, voire à la grand-mère. Elle devient ainsi la « mère », la « daronne » ou la « mémé » du président. On l'essentialise en « grand-maman Brigitte ", " mamie Trogneux », « mère Macron ». En ce qui concerne cette dernière expression, il est à souligner, comme le remarque L. Rosier (2017 : $11)$, que « [1] e syntagme Mère $+X$ déjà envisage pour des femmes de tous âges une disqualification signifiant l'autoritarisme, le caractère acariâtre d'une matrone [...]». De plus, mère Macron évoque mère maquerelle, expression avec l'emploi de laquelle « la vénalité de cette relation est soulignée » et «l'image d'un gigolo se profile ».

La critique du second type consiste à questionner la réalité du couple Macron. L'asymétrie générationnelle entre les époux est à l'encontre de la pensée dominante, des croyances et des valeurs partagées, ainsi suspectet-on des intérêts cachés dans cette relation : B. Macron doit forcément être une cougar. Ce terme désignant une femme mûre qui recherche et séduit des hommes beaucoup plus jeunes est une insulte explicite pour trois raisons au moins : il animalise la femme et la représente en prédatrice, il suggère une relation fausse (qui ne s'appuierait pas sur l'amour, mais sur l'intérêt) et il renforce l'asymétrie de traitement entre les deux $\operatorname{sex}^{4}{ }^{4}$, car il n'existe pas de terme spécifique pour désigner un homme en couple avec une femme plus jeune. Il faut aussi remarquer que qualifier $\mathrm{B}$. Macron de « cougar » revient à dire que son époux est un « gigolo » ou un « toy boy ». L'authenticité de l'amour d'un tel couple est contestée. Elle l'est aussi d'une autre manière : à un certain moment, le président s'est vu coller l'étiquette d'homosexuel, justement parce qu'il est doxiquement impensable qu'un jeune homme aime une femme beaucoup plus âgée. Il doit pour cela être un inverti qui se cache derrière une relation fausse parce que déplacée. Cette fausseté s'exprime dans le discours à travers les expressions telles que « couple d'opérette » (Anonyme, closermag.fr), « couple factice » (maminoucka, closermag.fr).

\subsection{Le couple devrait être basé sur la reproduction}

La sexualité inter-âge est diabolisée, car elle s'oppose au projet familial inscrit dans la doxa. L'épouse du président est stigmatisée pour son âge mûr qui exclut la possibilité de tomber enceinte, ce qui donne matière à des insultes implicites telles que celle lancée sur France Inter par l'humoriste Pierre-Emmanuel Barré, pour dire qu'il ne vote plus depuis longtemps : "Ma carte électorale, elle est comme la femme de Macron : ça fait longtemps qu'elle n'a plus de tampons ! ».

\footnotetext{
${ }^{4}$ En ce qui concerne la différence de traitement discursif des sexes, on peut se reporter à Yaguello (1978) ou à Houdebine-Gravaud (1995).
} 
C'est du même type d'humour douteux que fait preuve la une de Charlie Hebdo $\mathrm{du}$ 10.05.2017, où le nouveau président pose la main sur le ventre de sa femme enceinte, le tout épicé de la légende ironique Il va faire des miracles. Cette attaque vise non seulement le couple qui défie l'idée reçue selon laquelle la procréation devrait être son but majeur, mais surtout B. Macron qui s'écarte de l'archétype de l'épouse-mère.

\section{Les stéréotypes de la femme}

Le discours ciblant B. Macron dévoile aussi les idées reçues sur la femme qui imprègnent la société. Même si certains propos qui la concernent ne peuvent être considérés comme des insultes, on a très souvent affaire à des discours qui, consciemment ou non, présentent d'elle une vision discriminante et sexiste. Dans le discours analysé, deux d'entre les nombreux stéréotypes sautent aux yeux, l'un qui impose à la femme le rôle d'être jeune et jolie, l'autre opposant à l'activité masculine, sa passivité, sa dépendance, sa soumission et sa position en retrait de l'homme.

\subsection{Le rôle de la femme est d'être jeune et jolie}

Ce stéréotype constitue le soubassement doxique de tous les discours raillant l'âge et le physique de B. Macron. Cependant, il ne s'agit pas seulement de propos ouvertement injurieux. La doxa valorisant chez la femme la beauté physique se profile aussi, d'une manière moins sensible, mais tout aussi dangereuse, dans une presse contribuant largement à la construction médiatique d'une « féminité » réduite à l'aspect physique : on ne compte plus les articles parlant de la tenue vestimentaire de B. Macron, de ses jambes, de son look haute-couture. Il semble que le seul rôle assigné par la presse à Brigitte Macron soit le paraître. La presse ne la met en valeur que sous le prisme de l'habillement, et cette réduction à la seule apparence est en ellemême discriminante.

Or si B. Macron ne déroge pas au stéréotype d'un rôle décoratif auprès de son mari, elle ne s'inscrit pas dans la doxa qui valorise chez la femme la jeunesse. On l'attaque avec des insultes telles que « mamie », «monument historique » ou « momie », mais paradoxalement, on critique son allure jeune, ce qui trouve son plus haut degré d'expression dans l'insulte cagole. Ce terme désigne aujourd'hui une fille vulgaire qui se fait remarquer par une série de traits ostentatoires d'une féminité exacerbée. En appliquant ce terme à B. Macron, on fait ressortir la blondeur, le bronzage, la façon de s'habiller. Il s'agit bien évidemment d'une image outrancière, car elle n'est ni fille, ni vulgaire. 


\title{
2.2. La femme devrait être passive et soumise
}

La seconde image de la femme dans la doxa française qui lui impose passivité et soumission, est liée à la première : les deux se résument assez bien dans le fameux « Sois belle et tais-toi ». Bourdieu (1998: 73) le résume en ces mots :

\begin{abstract}
La domination masculine, qui constitue les femmes en objets symboliques, dont l'être (esse) est un être-perçu (percipi), a pour effet de les placer dans un état permanent d'insécurité corporelle ou, mieux, de dépendance symbolique : elles existent d'abord par et pour le regard des autres, c'est-à-dire en tant qu'objets accueillants, attrayants, disponibles. On attend d'elles qu'elles soient « féminines », c'est-à-dire souriantes, sympathiques, attentionnées, soumises, discrètes, retenues, voire effacées. [...] En conséquence, le rapport de dépendance à l'égard des autres (et pas seulement des hommes) tend à devenir constitutif de leur être.
\end{abstract}

Or le couple Macron semble être une union moderne dans laquelle la femme est placée au même niveau que l'homme : E. Macron a insisté lui-même à plusieurs reprises sur le fait que son épouse ne resterait pas au second plan. Effectivement, c'est une femme très active qui l'accompagne dans ses tâches, au point de se trouver souvent accusée d'influencer ses décisions.

L'activité et l'insoumission de la femme sont si fortement anti-doxiques qu'elles suggèrent a contrario la passivité de l'homme. Ainsi, les détracteurs d'E. Macron rappellent que l'épouse du président a été son professeur de théâtre et induisent que cette relation enseignant-élève persisterait dans leur mariage. L'image de professeur toujours prête à réprimander vise certes à discréditer B. Macron, mais surtout son époux. La critique du président passe par celle de sa femme qui brise les stéréotypes, démarche argumentative toute singulière que nous appelons une argumentation « ad coniugem ». Elle consiste, comme on le verra, à présenter E. Macron comme un incompétent que les choix amoureux disqualifient en tant que président de la République.

\section{La doxa et le discours argumentatif : stratégies et schèmes perceptifs}

Si les détracteurs d'E. Macron jugent profitable de recourir aux stéréotypes concernant sa relation amoureuse, c'est parce que, comme l'élucide Amossy $(1994: 47)$ :

La stéréotypie entendue au sens large du terme fournit son soubassement au discours argumentatif en le fondant sur une strate d'images et d'idées familières susceptibles de produire un effet d'évidence. C'est dire que la bonne marche de l'argumentation dépend en partie du maniement des idées reçues et des stéréotypes.

De plus, dans le cas qui nous occupe, il s'agit d'une situation compétitive, d'une polémique où s'affrontent les tenants d'options politiques différentes. Le discours des antagonistes d'E. Macron se fonde sur la doxa, non seulement parce 
que s'en réclamer permet de valider par avance les propos qui sont avancés sur le mode du « cela va sans dire », mais aussi parce que le partage des stéréotypes au sein d'une même communauté va de pair avec la construction d'un sentiment d'appartenance à cette communauté. Le public cible du discours attaquant le président est composé de ceux qui s'opposent à lui et peuvent ainsi maintenir la cohésion et la solidarité de leur groupe. Une vision du monde commune donne à ceux qui la partagent l'impression de constituer un ensemble homogène et solidaire qui a raison contre ceux qui pensent autrement.

Nous avons relevé dans le discours des antagonistes de Macron plusieurs schèmes perceptifs servant deux principales stratégies argumentatives : l'infantilisation et la dévirilisation. Parmi les images simplistes du président, construites invariablement à travers les références à son épouse, cinq reviennent de manière récurrente : mère + enfant, grand-mère + petit-fils, professeur + élève, pédophile + enfant harcelé et « couverture »+ homosexuel.

\subsection{Infantilisation}

L'âge de B. Macron est pour les détracteurs de son époux, prétexte à l'infantiliser : il est décrit soit comme un enfant, soit comme un homme atteint du complexe d'Edipe, ce qui implique que son épouse est sa mère. L'internet déborde de critiques qui se nourrissent de cette image, y compris celles de journalistes :

(1) Il a épousé sa prof et tué François Hollande ! Question Edipe, Emmanuel Macron est un sacré client pour le divan. Transgressif et ambivalent à souhait, le jeune président de la République, qui s'est érigé en totem en bousculant les tabous, a de quoi fasciner les analystes de toutes les chapelles.

nouvelobs.com, 10.10 .2018

Ce schème perceptif est mis à profit aussi dans la parole citoyenne, tant de manière explicite, qu'implicite, très souvent sur un ton ironique. Le président est présenté comme un enfant avec tout ce que cela suppose, parfois même comme un enfant harcelé par une pédophile (les Macron se sont connus et aimés quand Emmanuel avait 16 ans) :

(2) Toujours aussi méprisant du peuple français Oedipe se croit au dessus de tout

ArtCeltique sur lefigaro.fr, 28.09.2018

(3) [...] il n'est pas à la hauteur du poste qu'il vise le mignon... Maman le pousse en avant mais il a encore beaucoup de choses à apprendre... 
(4) Parlons peu mais bien... Lui c'est le toy boy et elle sa cougar... Une... Une... Pédophile, puisque elle a abusé d'un mineur à l'époque où ils se sont rencontrés. Lui étudiant mineur et elle prof de français avec 25 ans de plus ou davantage... Mais que s'est-il passé? Pourquoi les parents n'ont pas déposé plainte envers cette pédo-cougar?

Jev sur ripostelaique.com, 25.08.2017

L'énoncé (4) est d'ailleurs, on le voit, un exemple de commentaire accumulant plusieurs schèmes et autant d'insultes, comme si un(e) seul(e) n'était pas suffisant(e), selon l'argumentateur, pour discréditer la cible du discours. E. Macron est non seulement un enfant, mais encore un enfant victime d'abus sexuel, bien plus : à sa propre demande.

Il semble que la stratégie de délégitimation, mêlant les schèmes perceptifs et hyperbolisant à l'extrême l'anti-doxicité du couple Macron, soit caractéristique de l'argumentation ad personam, de même que tout procédé permettant de renforcer le message, telles l'accumulation, la répétition ou l'énumération :

(5) Enfant doublement " gâté »! Par son enfance, ses études, et les gâteries de la Brizzitte gâteuse, Par ses relations avec la Finance, le Mondialisme, Attali, Soros et Bilderberg...

Polyeucte sur ripostelaique.com, 2.06.2019

Ce commentaire argumente d'un côté par l'exagération, de l'autre, par la concision. L'énonciateur fait une référence hyperbolique à l'âge de la première dame (Brizzitte gâteuse) et à la puérilité supposée du président (enfant gâté), mais joue aussi sur la densité sémantique de la forme Brizzitte (que l'on peut rencontrer dans de nombreux commentaires sur la toile, et pas seulement sur le site du journal Riposte laïque, condamné à plusieurs reprises pour incitation à la haine) accentuant à l'extrême le léger zozotement d'E. Macron et faisant une allusion méchante à son image d'éternel enfant.

Le schème perceptif grand-mère + petit-fils intensifie plus encore la stratégie d'infantilisation, diabolisant l'écart d'âge entre les époux. En témoignent les deux énoncés qui suivent, mais la stigmatisation dudit écart atteint son paroxysme dans le commentaire (7) dont l'auteur présente le président comme un nourrisson ne maitrisant pas encore ses fonctions naturelles et sa femme comme une vieille ne les maîtrisant plus :

(6) Les rencontres de notre minable minet avec Poutine ou Trump ont démontré que la France n'était pas représentée par un PR mais par un gringalet, aux pellicules, accroché aux jupons de sa grand mère.

Désespoir50 sur dreuz.info, 28.07.2018

(7) [...] Peut-être un peu au bord de la bienséance... une couche pour l'Enfant roi et une couche pour Bizitte !!! 
Les posts disqualifiant le chef d'État à l'aide du schème perceptif professeur + élève ne cadrent point avec la situation qui les suscite ; on voit là le propre de l'argumentation ad personam. Ainsi, quand E. Macron, alors encore candidat à l'élection, a commis une erreur en évoquant « l'île » de Guyane, une vague de commentaires moqueurs a déferlé :

(8) pauvre macron il ne connaît même pas sa géographie, ce n'est pas la peine d'avoir coucher avec sa prof

Anonyme sur closermag.fr, 27.03.2017

(9) macron demande à mamie de te donner des cours de géo. lol

Anonyme sur closermag.fr, 27.03.2017

(10) Brigitte, il faudra lui imposer de sacrées révisions au jeunot

Hou le villain sur lefigaro.fr, 28.03.2017

\subsection{Dévirilisation}

E. Macron est non seulement infantilisé, mais aussi dévirilisé. On le présente comme un inverti et sa femme comme une « couverture » de son homosexualité :

(11) Brigitte n'est qu'une couverture ridée pour ses gay pride...

particeesnault sur twitter.com, 8.04.2017

(12) Etant entouré de « Tafioles » et cornaqué par les dites « Tafioles » comment voulez vous qu'il n'en soit pas une ? Sa cougar n'est qu'une baudruche représentative de loin, car de près on dirait la momie de Ramses II.

Zorglub sur ripostelaique.com, 26.08.2017

Certains commentaires misent aussi sur l'implicite :

(13) Grand diseux, petit faiseux, dit avec raison le dicton.

mistophore sur madame.lefigaro.fr, 28.04.2017

Puisqu'il est fait référence au candidat à la présidentielle, on pourrait interpréter ce message comme commentant ses discours jugés creux ou ses promesses qui ne vont jamais être réalisées, mais ce commentaire est une réaction à l'article paru sur madame.lefigaro.fr intitulé " L'atypique couple Macron fascine les Chinois » (28.04.2017). Le contexte permet donc d'inférer ce dont l'argumentateur tente de persuader le public : l'asymétrie générationnelle entre les époux Macron donne à penser : ne s'agirait-il que d'intérêts cachés dans ce " couple» ? Il s'appuie pour cela sur la doxa ambiante, en vertu de laquelle la femme ne peut être désirable que 
jusqu'à un certain âge. On valorise les hommes qui ont su conquérir une femme plus jeune, E. Macron doit donc être un homosexuel. Comme le souligne Amossy (1999: 82), l'implicite peut être le meilleur « allié » de l'argumentation grâce à la connivence qu'il permet de créer :

Dans l'allusion, qui est une variété du sous-entendu, il est fait référence à quelque chose qui n'apparaît pas dans la littéralité du discours, qui est connu de certains interactants et d'eux seuls, et qui, de ce fait, établit entre eux une connivence. L'allusion emporte d'autant mieux l'adhésion de son public de prédilection qu'elle lui donne le sentiment d'appartenir au groupe des élus.

\section{Conclusion}

L'image qui émerge du discours attaquant le président à travers sa relation conjugale est celle d'un gamin ou d'un inverti, et ce, par le seul fait qu'il a osé contrarier la doxa et briser les tabous sociaux en se mariant avec une femme de 24 ans son aînée et, de plus, son professeur. On présente ce fait comme l'un des défauts qui le discréditent comme président, ce qui relève sans doute d'une argumentation fallacieuse, car évoquer les aspects de sa vie privée pour le délégitimer comme homme politique, n'est pas une démarche persuasive raisonnable ni honnête. L'argument de l'écart d'âge entre époux ne sert pas à rechercher la vérité ou à faire une critique constructive de la politique de Macron, mais à le présenter d'emblée comme puéril, soumis, incapable de gérer le pays.

Cependant, s'il est vrai que les arguments ad personam sont abusifs, les références constantes à l'épouse d'E. Macron paraissent compréhensibles : le président assume pleinement l'écart d'âge qui les sépare en plaçant sa femme sur le devant de la scène. On peut interpréter son attitude comme une argumentation propre à lui : qu'il soit capable de former un couple anti-doxique est un argument en sa faveur, parce qu'il désire se présenter comme incarnant le renouveau et la modernité. Il construit son éthos prédiscursif d'un président moderne défiant les convenances, conscient peut-être du fait que la doxa s'oppose à la nouveauté pour procurer un confort mental à la communauté qui n'aime pas les changements.

\section{Bibliographie}

AMOSSY, Ruth (1994), « Stéréotypie et argumentation » in Le Stéréotype. Crise et transformations (A. Goulet dir.), Caen, Presses universitaires de Caen, p. 47-61

AMOSSY, Ruth (1999), «Israël et les juifs dans l'argumentation de l'extrême droite : doxa et implicite », Mots. Les langages du politique, $\mathrm{n}^{\circ}$ 58, p. 79-100

AMOSSY, Ruth (2006 [2000]), L'Argumentation dans le discours, Paris, Armand Colin

AMOSSY, Ruth, HERSCHBERG PIERROT, Anne (1997), Stéréotypes et clichés : langue, discours, société, Paris, Nathan 
ANGENOT, Marc (2013), Rhétorique de la confiance et de l'autorité, Montréal, Discours social BOURDIEU, Pierre (1998), La domination masculine, Paris, Seuil

BOZON, Michel (1990), «Les femmes et l'écart d'âge entre conjoints : une domination consentie », Population, $\mathrm{n}^{\circ}$ 2, année 45 , p. 327-360

BOZON, Michel (1991), «Les femmes plus âgées que leur conjoint sont-elles atypiques ?", Population, $\mathrm{n}^{\circ}$ 1, année 46, p. 152-159

COENEN-HUTHER, Josette (2001), « Dominance et égalité dans les couples », Cahiers du genre, $\mathrm{n}^{\circ} 30$, p. $179-204$

GAUTHIER, Gilles (2011), « L'argument ad hominem en communication politique » in L'Argumentation (N. D'Almeida coord.), Paris, CNRS Éditions, p. 77-95

HARDING, John (1968), "Stereotypes » in International Encyclopedia of the Social Sciences (D. L. Sills éd.), vol. 15, New York, The McMillan Cie \& The Free Press, p. 259-262

HOUDEBINE-GRAVAUD, Anne-Marie (1995), « Des femmes dans la langue et les discours ", Cahiers des Annales de Normandie, $\mathrm{n}^{\circ}$ 26, p. 385-398

MIGNOT, Jean-François (2010), «L'écart d'âge entre conjoints », Revue française de sociologie, $n^{\circ} 51 / 2$, p. $281-320$

ROSIER, Laurence (2017), De l'insulte ... aux femmes, Bruxelles, $180^{\circ}$ éditions

SCHAPIRA, Charlotte (1999), Les stéréotypes en français. Proverbes et autres formules, Paris, Ophrys

SLAKTA, Denis (1994), "Stéréotype : sémiologie d'un concept » in Le Stéréotype. Crise et transformations (A. Goulet dir.), Caen, Presses universitaires de Caen, p. 35-45

YAGUELLO, Marina (1978), Les mots et les femmes. Essai d'approche socio-linguistique de la condition féminine, Paris, Payot

Agnieszka Konowska - maître de conférences à l'Institut d'Études romanes de l'Université de Łódź (Pologne), docteur en sciences humaines (spécialité : linguistique). Auteur et co-rédacteur de quatre monographies et d'une trentaine d'articles scientifiques. Ses principaux domaines d'intérêt scientifique sont la pragmatique linguistique, l'analyse argumentative du discours et la stéréotypie discursive. Ses recherches actuelles portent principalement sur le fonctionnement argumentatif des noms propres dans le discours. 